\title{
Intensity Dependence of Photoassociation in a Quantum Degenerate Atomic Gas
}

\author{
Ionut D. Prodan, Marin Pichler, Mark Junker, and Randall G. Hulet \\ Department of Physics and Astronomy and Rice Quantum Institute, Rice University, Houston, Texas 77251, USA \\ John L. Bohn \\ JILA, National Institute of Standards and Technology and University of Colorado, Boulder, Colorado 80309-0440, USA
}

(Received 9 July 2002; published 19 August 2003)

\begin{abstract}
We have measured the intensity dependent rate and frequency shift of a photoassociation transition in a quantum degenerate gas of ${ }^{7} \mathrm{Li}$. The rate increases linearly with photoassociation laser intensity for low intensities, whereas saturation is observed at higher intensities. The measured rates and shifts agree reasonably well with theory within the estimated systematic uncertainties. Several theoretically predicted saturation mechanisms are discussed, but a theory in which saturation arises because of quantum mechanical unitarity agrees well with the data.
\end{abstract}

DOI: $10.1103 /$ PhysRevLett.91.080402

Photoassociation (PA) of ultracold atoms has been a remarkably useful tool for determining scattering lengths characterizing ultracold atom collisions, for producing ultracold molecules, and for providing extremely precise measurements of atomic radiative lifetimes (see Refs. [1,2] for reviews). This utility is largely a consequence of the spectroscopic precision afforded by the small thermal broadening in a laser or evaporatively cooled gas. Quantum degenerate gases are especially interesting because the coherence of the atomic field may enable the formation of a molecular Bose-Einstein condensate (BEC) from an atomic one by coherent Raman transitions [3,4], stimulated Raman adiabatic passage [5], or by other coherent adiabatic population transfer schemes $[6,7]$.

There have been extensive theoretical studies of the rate of PA [8-12]. The rate is predicted to increase linearly with intensity at low intensities, while various mechanisms have been proposed that cause saturation of the rate at higher intensities. Among these mechanisms are the quantum mechanical unitarity limit on the rate of atomic collisions [11], a breakdown of the two-mode approximation $[4,13]$ for PA of Bose condensates caused by coupling to noncondensed atomic modes [12,14-16], and the depletion of the atomic pair correlation function [15]. Photoassociation resonances are also predicted to exhibit a spectral shift proportional to the light intensity caused by coupling to the continuum of free-atom states [11,17-19]. In contrast to theory, there are relatively few experimental measurements, and only two that could be considered "precise" (which we define as measurements with uncertainties of less than a factor of 2). We previously measured the spectral light shift using quantum degenerate ${ }^{7} \mathrm{Li}$ and obtained good agreement with theory [20]. Both the spectral shift and the PA rate constant were recently measured in a $\mathrm{Na}$ condensate, and good agreement with two-body theory was found [21]. Saturation was not observed in this experiment. Saturation was observed in two other lower precision experi-
PACS numbers: 03.75.Nt, 33.20.Kf, 33.70.-w, 34.20.Cf

ments [22,23], but these experiments were performed in a magneto-optical trap, where the temperatures were greater than $100 \mu \mathrm{K}$ and the corresponding unitaritylimited rates were quite small, of the order of $1 \mathrm{~s}^{-1}$ or less.

We report precise measurements of both the rate of PA and the spectral shift in a quantum degenerate gas of ${ }^{7} \mathrm{Li}$ atoms as a function of laser intensity. The rate is observed to saturate at the highest intensities, where the corresponding rate constants are nearly 2 orders of magnitude larger than any previously measured. The large PA rate is a consequence of quantum degeneracy, where the densities are relatively high and the unitarity-limited rates are large, as well as favorable free-bound state overlap in lithium.

The apparatus will be discussed only briefly as it has been described in detail previously [20,24]. Atoms are confined by a magnetic trap with a bias field of $\sim 1000 \mathrm{G}$. Atoms in the $F=2, m_{F}=2$ hyperfine sublevel of ${ }^{7} \mathrm{Li}$ are cooled by evaporation to quantum degeneracy. Attractive interactions between atoms restrict the number of atoms in the Bose-Einstein condensate, $N_{0}$, to a relatively small number before the condensate collapses [25]. For the experimental conditions here, $N_{0} \lesssim 1250$, a small fraction of the total number of atoms. This fact, however, facilitates the achievement of repeatable temperatures, total atom numbers, and densities because quenching the gas below the BEC transition temperature $T_{c}$ causes it to attain thermal equilibrium with a temperature $T \simeq$ $T_{c}$ [26]. Therefore, following evaporation we allow the gas to equilibrate for approximately $12 \mathrm{~s}$, at which point the total number of atoms $N \simeq 8 \times 10^{5}$, the gas is very near the $\mathrm{BEC}$ transition at $T \simeq 600 \mathrm{nK}$, and the peak density is $\sim 4 \times 10^{12} \mathrm{~cm}^{3}$.

Following equilibration, the atoms are exposed to a pulse of photoassociation light. Up to $85 \mathrm{~mW}$ of PA light, coupled out of a single-mode fiber is focused onto the atoms. The laser frequency is tuned to near resonance with the $v^{\prime}=83$ vibrational level of the $1^{3} \Sigma_{g}^{+}$state, 
which has a binding energy of $60 \mathrm{GHz}$ relative to the $2 P_{1 / 2}$ asymptote [27]. The relative frequency of the PA laser is monitored by comparing it with a reference laser locked to an atomic transition using a scanning FabryPerot etalon. The $v^{\prime}=83$ level was chosen for its large ratio of the photoassociation rate to the rate of offresonant scattering from the atomic resonance.

Photoassociation can be detected because it causes a reduction in atom number when excited molecules spontaneously decay into pairs of hot atoms that escape the trap [1,2]. The number of atoms remaining following the PA pulse is determined by polarization phase-contrast imaging [24], and compared with a "background" measurement of the number of atoms without the PA pulse. Since imaging destroys the sample, the trap must be reloaded and the atoms evaporatively cooled for each image. Both $N$ and $T$ are extracted by fitting the images to the distribution function for a Bose gas in an external potential. The photoassociation rate is determined for a given laser intensity $I$ by adjusting the laser pulse length $\tau$ to cause a $20 \%-30 \%$ loss of the initial number of atoms. For the data presented here, $\tau$ is between $12 \mu \mathrm{s}$ at the highest intensity and $4 \mathrm{~ms}$ at the lowest. Other phenomena besides PA, such as spontaneous scattering from the atomic resonance and dipole forces, can cause lightinduced trap losses. Dipole forces, arising from gradients of the laser field, are minimized by using a large laser beam waist $\left(1 / e^{2}\right.$ radius of between 220 and $\left.320 \mu \mathrm{m}\right)$. The background loss rate is measured by tuning the PA laser several $\mathrm{GHz}$ from the molecular resonance, and the data are adjusted accordingly, by up to $2 \%$ at the highest intensities. Figure 1 shows a typical photoassociation resonance curve for relatively low intensity. The curve is well described by a Lorentzian with a width only slightly larger than the expected value of twice the atomic natural linewidth. Although we did not perform a systematic study of line shapes, the resonance broadens as expected as $I$ is increased. The resonance position shifts with laser intensity, as shown in Fig. 2. A linear fit to the data gives a slope of $(-1.7 \pm 0.2) \mathrm{MHz} /\left(\mathrm{W} \mathrm{cm}^{-2}\right)$.

The measurement of the rate is expressed in terms of the on-resonance rate coefficient $K_{p}$, which is defined in terms of the time derivative of the density $\dot{n}(\mathbf{r}, t)=$ $-K_{p}(I) n^{2}(\mathbf{r}, t)$. We neglect the effect of elastic collisions on the density distribution since $\tau$ is much less than the characteristic time for elastic scattering of $\sim 300 \mathrm{~ms}$. The solution for the evolution of the density, $n(\mathbf{r}, t)=$ $n(\mathbf{r}, 0) /\left\{1+K_{p}(I) n(\mathbf{r}, 0) t\right\}$, shows that the distribution becomes flatter during the PA pulse since the rate is largest at the highest densities [21]. The initial density $n(\mathbf{r}, 0)$ prior to the PA pulse is described by an equilibrium Bose-Einstein density distribution. The fractional trap loss, for a given value of $K_{p}$ and $\tau$, is calculated by taking the ratio between the spatial integral of $n(\mathbf{r}, \tau)$ and the initial number of atoms. The best value for $K_{p}$ is found numerically by matching the computed fractional loss with the measured one. Figure 3 shows the dependence

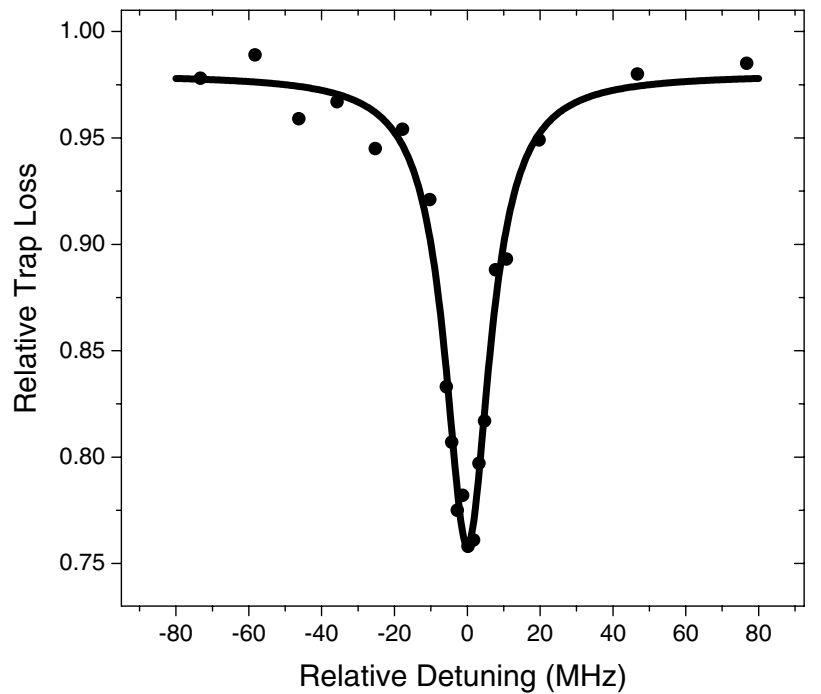

FIG. 1. Photoassociation resonance curve for $v^{\prime}=83$. The solid circles are experimental data points and the solid line is a Lorentzian fit to them. The signal is the normalized number of atoms remaining in the trap after the photoassociation pulse of intensity $0.185 \mathrm{~W} / \mathrm{cm}^{2}$ and duration $2.0 \mathrm{~ms}$. The initial number of atoms is $5 \times 10^{5}$ at a temperature of $\sim 520 \mathrm{nK}$. The uncertainty in relative frequency is $\sim 2 \mathrm{MHz}$, caused by drift of the PA and reference laser frequencies. The data fit to a rate constant with a Lorentzian width of $14 \mathrm{MHz}$, in good agreement with the expected natural width of 11.7 MHz, which is twice the atomic natural width [10]. The thermal contribution to the line shape is negligible at this temperature.

of the on-resonance rate coefficient $K_{p}$ on the laser intensity $I$. In the low-intensity limit $K_{p}$ is found to depend linearly on the laser intensity, with a slope of $(7.9 \pm$ 1.6) $\times 10^{-10}\left(\mathrm{~cm}^{3} \mathrm{~s}^{-1}\right) /\left(\mathrm{W} \mathrm{cm}^{-2}\right)$. The rate constant saturates at high intensities, with a maximum value of $(2.2 \pm 0.2) \times 10^{-8} \mathrm{~cm}^{3} \mathrm{~s}^{-1}$. The stated uncertainties are dominated by systematic effects.

Several factors contribute to the uncertainties of the measured quantities. There is no direct way to know $N$ before exposure to the PA pulse, and therefore the ability to measure PA-induced loss depends on the reproducibility of $N$ and $T$. We find that the statistical variations in $N$ and $T$ for the background measurements have standard deviations of only $3 \%$ and $2 \%$, respectively. Furthermore, the phase-space density, proportional to $N T^{-3}$, is found to be within $5 \%$ of the critical value for all background measurements. Background measurements are typically made every fourth shot to monitor and make adjustments for long-term drifts. Of more concern are systematic uncertainties. $N$ and $T$ are subject to systematic uncertainties of $5 \%$ and $3 \%$, respectively, due to uncertainties in the image magnification and probe laser polarization [24]. Finally, the uncertainty in intensity is dominated by a systematic uncertainty in power measurement, which we estimate to be $\sim 10 \%$. These effects combine to give a $20 \%$ uncertainty to the slope of $K_{p}$ and a $10 \%$ uncertainty 


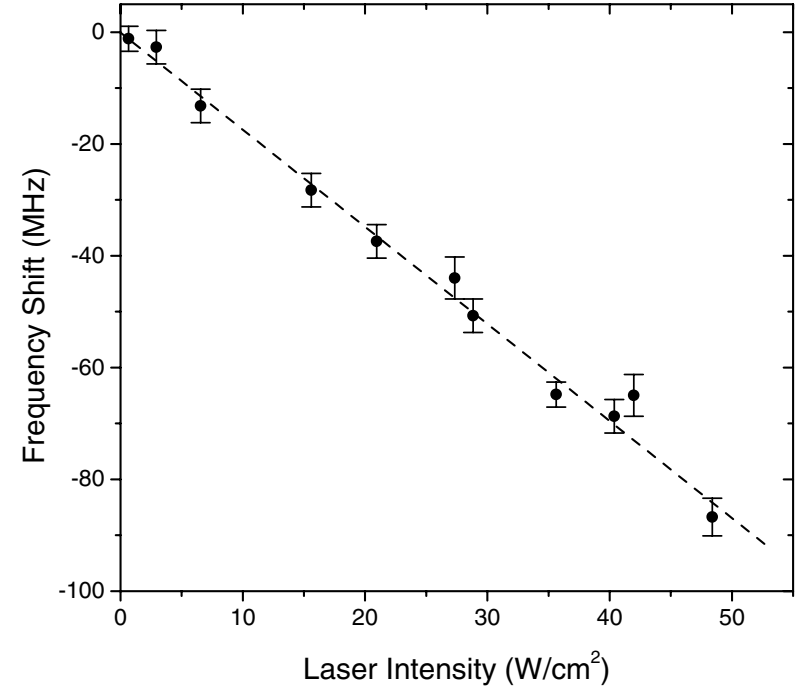

FIG. 2. Light-induced frequency shift of the photoassociation resonance. The solid circles are data. The error bars are uncertainty estimates for the identification of the resonance peak positions. The dashed line is a fit to a straight line, where each data point is weighted by the inverse of the error bar length.

to both the rate constant maximum and the slope of the frequency shift.

The data are compared with the theory of Ref. [11]. The transition dipole, $d_{m}$, for the free-bound transition to this high-lying vibrational level is simply related to the atomic dipole $d_{a}$. Since the binding energy of $v^{\prime}=83$ is large compared to the fine-structure splitting of the $2 P$

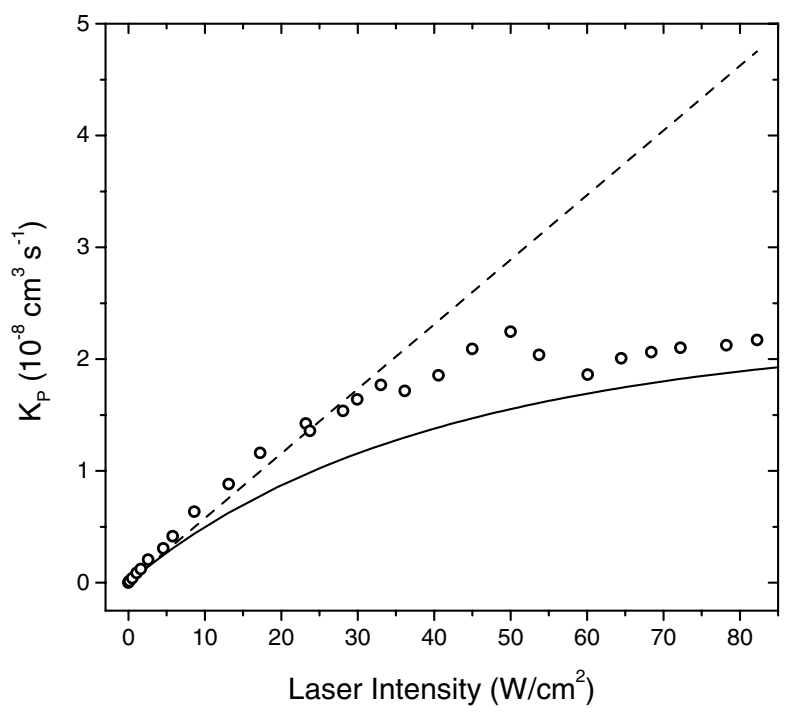

FIG. 3. On-resonance photoassociation rate coefficient $K_{p}$ as a function of laser intensity. The open circles are experimental data, obtained from trap loss analysis. The solid line is the theoretical prediction, with no adjustable parameters. The dashed line is an extrapolation of the theoretical result in the low-intensity limit. atomic state and any hyperfine interactions, the electronic and nuclear spins of the atoms decouple from the PA light field. Therefore, for this $\Sigma \rightarrow \Sigma$ transition, $d_{m}$ lies along the internuclear axis. Averaging over the unknown orientation of the molecular axis relative to the polarization axis of the linearly polarized laser field gives a factor of $\sqrt{1 / 3}$, so that $d_{m}=\sqrt{2 / 3} d_{a}$ [28]. The free-bound FranckCondon factors are numerically evaluated using molecular potentials determined previously [29,30]. Finally, because of quantum degeneracy, to calculate $K_{p}$ we average the scattering matrix element over a Bose-Einstein energy distribution.

The light-induced spectral shift is calculated to be $-2.1 \mathrm{MHz} /\left(\mathrm{W} \mathrm{cm}^{-2}\right)$, in reasonably good, but not perfect agreement with the measured value. The solid line in Fig. 3 shows the theoretical results for $K_{p}$. The dashed line is a low-intensity extrapolation of the theory which has a slope of $5.8 \times 10^{-10}\left(\mathrm{~cm}^{3} \mathrm{~s}^{-1}\right) /\left(\mathrm{W} \mathrm{cm}^{-2}\right)$, which again is in reasonable agreement. Finally, theory predicts saturation to $K_{p}=2.0 \times 10^{-8} \mathrm{~cm}^{3} \mathrm{~s}^{-1}$, which agrees very well with the measured value.

The theory of Refs. [8,9] show that $K_{p}$ goes to a constant value, independent of $T$, in the low- $T$ and low- $I$ limits. However, the free-bound matrix element depends on $T$ in this theory. We find it convenient to define the free-bound matrix element as $T$ independent from the start, and use this to obtain a completely $T$-independent analytic approximation for $K_{p}$. Therefore, we scale the two-atom unbound wave function $\phi_{k}(r)$ such that outside of the interior of the ground-state molecular potential $\phi_{k}(r)=\frac{\sin (k r+\delta)}{k r}$, where $\delta$ is the $s$-wave scattering phase shift. The overlap between the unity-normalized molecular wave function $\psi_{v}(r)$ and $\phi_{k}(r)$ is $M_{k v}=$ $4 \pi \int_{0}^{\infty} r^{2} \phi_{k}(r) \psi_{v}^{*}(r) d r$. This matrix element has units of (length) $)^{3 / 2}$ and reaches a constant value as $T \rightarrow 0$. In our experiment, for example, at $T \simeq 600 \mathrm{nK}$ and for $v^{\prime}=83$, the wave number $k \simeq 2 \times 10^{-4} a_{o}^{-1}$, the classical outer turning point $R_{c}=103 a_{o}$, and the $s$-wave scattering length $a=-27.6 a_{o}$ [29], where $a_{o}$ is the Bohr radius. The value of $\left|M_{k v}\right|^{2}$ for these conditions differs from the $T \rightarrow 0$ value by only $0.02 \%$, showing that the system is well within the quantum threshold regime. At low intensities and temperatures, we find $K_{p}=\gamma \frac{I}{I_{\mathrm{sat}}}\left|M_{k v}\right|^{2}$, where $I_{\text {sat }}$ and $\gamma$ are the molecular saturation intensity and spontaneous decay rate, respectively. For this transition $I_{\text {sat }}=6 I_{a}$, where $I_{a}=5.1 \mathrm{~mW} / \mathrm{cm}^{2}$ is the atomic saturation intensity.

Saturation comes about in the theory of Ref. [11] because of the quantum mechanical unitarity limit on the rate of two-body collisions. Accordingly, the upper bound for the rate constant is $K_{p}^{(u)}=\sigma\langle v\rangle$, where $\sigma=\frac{2}{\pi} \lambda^{2}$ is the maximum cross section between identical, noncondensed bosons, $\langle v\rangle$ is the thermally averaged velocity, and $\lambda$ is the thermal de Broglie wavelength. For $T=600 \mathrm{nK}, K_{p}^{(u)}=$ $2.5 \times 10^{-8} \mathrm{~cm}^{3} \mathrm{~s}^{-1}$, in good agreement with the full theoretical calculation and experiment. The saturation observed in previous experiments [22,23] is consistent 
with the unitarity limit, although at rates many orders of magnitude lower than observed here. It is interesting to speculate on how our results relate to the other proposed saturation mechanisms. The reverse process of dissociation can result in the formation of pairs of "hot" atoms that are not returned to the original translational state of the cold atomic gas [12,14-16]. The energy width of the dissociated pairs is proportional to the PA rate, and results in a rate limit of $\sim \hbar n^{2 / 3} / m$ [16]. Interestingly, at $T_{c}$, where $\lambda \propto n^{-1 / 3}$, the unitarity limit gives the same maximum rate $n K_{p}^{(u)} \simeq \hbar n^{2 / 3} / \mathrm{m}$. Since association must involve atom pairs in close proximity, depletion of the pair correlation function at short range may also limit the rate. From a classical, particlelike perspective, the maximum association rate is reached when atoms cannot move quickly enough to replenish the necessary close-range pairs, leading to a maximum rate of $\sim v / d$, where $d \simeq$ $n^{-1 / 3}$ is the mean separation between atoms and $v$ is their mean velocity. But since $v \simeq \hbar / m \lambda$ and $n \simeq N_{0} / \lambda^{3}$, the resulting limit is $\sim N_{0}^{-1 / 3} \hbar n^{2 / 3} / m$, which is again the same as the unitarity limit when $T \simeq T_{c}$. The limit imposed by this classical argument, however, has already been violated in the BEC experiment reported in Ref. [21], in which a PA rate greatly in excess of $v / d$ was observed. Although no saturation was observed in that experiment, the rate was well below the unitarity limit, and $\hbar n^{2 / 3} / \mathrm{m}$. Our experiment cannot clearly distinguish between the mechanisms because their predicted limits are all of the same order at $T_{c}$. However, the overall good quantitative agreement over a large range of intensity with a theory whose rate is limited by unitarity in the two-body scattering amplitude, strongly supports this explanation for our data. Finally, we point out that mechanisms limiting the PA rate will also limit the rate of molecule formation by magnetic Feshbach resonances.

In summary, we report the results of precise measurements of the rate and spectral shift of photoassociation resonances of a quantum degenerate gas. The overall agreement between theory, with no free parameters, and our measurement is good. The agreement attests to the general validity of the theory and also points to quantum mechanical unitarity as the limitation of the rate for a gas at $T_{c}$. The distinction between the unitarity-limited model and the hot dissociation model could nevertheless be unambiguously tested with a lithium gas cooled significantly below $T_{c}$, an experiment we hope to perform in the future.

We thank Robin Côté, Juha Javanainen, and Paul Julienne for helpful discussions. This work was partially funded by grants from the National Science Foundation, the Office of Naval Research, NASA, and the Welch Foundation.

[1] J. Weiner, V. Bagnato, S. Zilio, and P. S. Julienne, Rev. Mod. Phys. 71, 1 (1999).
[2] W. C. Stwalley and H. Wang, J. Mol. Spectrosc. 195, 194 (1999).

[3] P. S. Julienne, K. Burnett, Y. B. Band, and W. C. Stwalley, Phys. Rev. A 58, R797 (1998).

[4] D. J. Heinzen, R. Wynar, P. D. Drummond, and K.V. Kheruntsyan, Phys. Rev. Lett. 84, 5029 (2000).

[5] M. Mackie, R. Kowalski, and J. Javanainen, Phys. Rev. Lett. 84, 3803 (2000).

[6] J. Javanainen and M. Mackie, Phys. Rev. A 59, R3186 (1999).

[7] F. H. Mies, E. Tiesinga, and P. S. Julienne, Phys. Rev. A 61, 022721 (2000).

[8] R. Napolitano, J. Weiner, C. J. Williams, and P.S. Julienne, Phys. Rev. Lett. 73, 1352 (1994).

[9] P. Pillet, A. Crubellier, A. A. Bleton, O. Dulieu, P. Nosbaum, I. Mourachko, and F. Masnou-Seeuws, J. Phys. B 30, 2801 (1997).

[10] R. Côté and A. Dalgarno, Phys. Rev. A 58, 498 (1998).

[11] J. L. Bohn and P.S. Julienne, Phys. Rev. A 60, 414 (1999).

[12] M. Koštrun, M. Mackie, R. Côté, and J. Javanainen, Phys. Rev. A 62, 063616 (2000).

[13] E. Timmermans, P. Tommasini, M. Hussein, and A. Kerman, Phys. Rep. 315, 199 (1999).

[14] K. Goral, M. Gajda, and K. Rzazewski, Phys. Rev. Lett. 86, 1397 (2001).

[15] M. Holland, J. Park, and R. Walser, Phys. Rev. Lett. 86, 1915 (2001).

[16] J. Javanainen and M. Mackie, Phys. Rev. Lett. 88, 090403 (2002).

[17] P. O. Fedichev, Y. Kagan, G.V. Shlyapnikov, and J.T. M. Walraven, Phys. Rev. Lett. 77, 2913 (1996).

[18] J. L. Bohn and P.S. Julienne, Phys. Rev. A 56, 1486 (1997).

[19] J. Javanainen and M. Mackie, Phys. Rev. A 58, R789 (1998).

[20] J. M. Gerton, B. J. Frew, and R. G. Hulet, Phys. Rev. A 64, 053410 (2001).

[21] C. McKenzie et al., Phys. Rev. Lett. 88, 120403 (2002).

[22] C. Drag, B. Tolra, O. Dulieu, D. Comparat, M. Vatasescu, S. Boussen, S. Guibal, A. Crubellier, and P. Pillet, IEEE J. Quantum Electron. 36, 1378 (2000).

[23] U. Schlöder, C. Silber, T. Deuschle, and C. Zimmermann, Phys. Rev. A 66, 061403 (2002).

[24] C. A. Sackett, C. C. Bradley, M. Welling, and R. G. Hulet, Appl. Phys. B 65, 433 (1997).

[25] C. C. Bradley, C. A. Sackett, and R. G. Hulet, Phys. Rev. Lett. 78, 985 (1997).

[26] C. A. Sackett, J. M. Gerton, M. Welling, and R. G. Hulet, Phys. Rev. Lett. 82, 876 (1999).

[27] E. R. I. Abraham, N.W. M. Ritchie, W. I. McAlexander, and R. G. Hulet, J. Chem. Phys. 103, 7773 (1995).

[28] In Ref. [20], we had erroneously assumed that $d_{m}$ was quantized along the magnetic field direction, and considering laser polarization, overestimated $d_{m}$ by a factor of $\sqrt{2}$.

[29] E. R. I. Abraham, W. I. McAlexander, C. A. Sackett, and R. G. Hulet, Phys. Rev. Lett. 74, 1315 (1995).

[30] W. I. McAlexander, E. R. I. Abraham, N.W. M. Ritchie, C. J. Williams, H. T. C. Stoof, and R. G. Hulet, Phys. Rev. A 51, R871 (1995). 